\title{
ON INVERSE SPECTRAL PROBLEM AND GENERALIZED STURM NODAL THEOREM FOR NONLINEAR BOUNDARY VALUE PROBLEMS
}

\author{
Ya. IL'YASOV, N. VALEEV
}

\begin{abstract}
In the present paper, we are concerned with the Sturm-Liouville operator$$
\mathcal{L}[q] u:=-u^{\prime \prime}+q(x) u
$$

subject to the separated boundary conditions. We suppose that $q \in L^{2}(0, \pi)$ and study a so-called inverse optimization spectral problem: given a potential $q_{0}$ and a value $\lambda_{k}$, where $k=1,2, \ldots$, find a potential $\hat{q}$ closest to $q_{0}$ in the norm of $L^{2}(0, \pi)$ such that the value $\lambda_{k}$ coincides with $k$-th eigenvalue $\lambda_{k}(\hat{q})$ of the operator $\mathcal{L}[\hat{q}]$.

In the main result, we prove that this problem is related to the existence of a solution to a boundary value problem for the nonlinear equation

$$
-u^{\prime \prime}+q_{0}(x) u=\lambda_{k} u+\sigma u^{3}
$$

with $\sigma=1$ or $\sigma=-1$. This implies that the minimizing solution of the inverse optimization spectral problem can be obtained by solving the corresponding nonlinear boundary value problem. On the other hand, this relationship allows us to establish an explicit formula for the solution to the nonlinear equation by finding the minimizer of the corresponding inverse optimization spectral problem. As a consequence of this result, a new method of proving the generalized Sturm nodal theorem for the nonlinear boundary value problems is obtained.
\end{abstract}

Keywords: Sturm-Liouville operator, inverse optimization spectral problem, nodal theorem for the nonlinear boundary value problems.

Mathematics Subject Classification: 34L05, 34L30, 34A55

\section{INTRODUCTION}

In the present paper, we are concerned with the relations between the existence of solutions to the so called inverse optimization spectral problem ([5, 6]) for the Sturm-Liouville operator

$$
\mathcal{L}[q] u:=-u^{\prime \prime}+q(x) u
$$

subject to the separated boundary conditions

$$
\begin{aligned}
& u(0) \cos \alpha+u^{\prime}(0) \sin \alpha=0, \\
& u(\pi) \cos \alpha+u^{\prime}(\pi) \sin \alpha=0,
\end{aligned}
$$

and the existence of weak solutions to the nonlinear boundary value problems

$$
\left\{\begin{array}{c}
-u^{\prime \prime}+q_{0}(x) u=\lambda u+\delta u^{3}, \quad x \in(0, \pi), \\
u(0) \cos \alpha+u^{\prime}(0) \sin \alpha=0, \\
u(\pi) \cos \alpha+u^{\prime}(\pi) \sin \alpha=0,
\end{array}\right.
$$

with $\delta=1$ and $\delta=-1$.

The second author was partially supported by RFBR grant no. 18-51-06002 Az-a. 
We suppose that $q \in L^{2}:=L^{2}(0, \pi)$. Under these conditions $\mathcal{L}[q]$ defines a self-adjoint operator on the Hilbert space $L^{2}(0, \pi)$ (see, e.g., [3, 8, 10]), so that its spectrum consists of an infinite sequence of eigenvalues $\sigma_{p}(\mathcal{L}[q]):=\left\{\lambda_{i}(q)\right\}_{i=1}^{\infty}$ which can be ordered as follows: $\lambda_{1}(q)<\lambda_{2}(q)<\ldots$. Furthermore, to each eigenvalue $\lambda_{k}(q)$, there corresponds a unique (up to a normalization constant) eigenfunction $\phi_{k}(q)$ with exactly $k-1$ zeros in $(0, \pi)$.

The inverse spectral problem consisting in recovering of the potential $q(x)$ from a knowledge of the spectral data is a classical problem and, beginning with the celebrated papers by Ambartsumyan [1] in 1929, Borg in 1946 [2], Gel'fand \& Levitan [4] in 1951, it received a lot of attention.

It is well known (see, e.g., [2, 4]) that the inverse spectral problem with only finitely many given eigenvalues $\left\{\lambda_{i}\right\}_{i=1}^{m}, m<+\infty$ have infinitely many solutions and in general is meaningless. However, if one assume that a certain information about the potential $q$ is known in advance, for instance, an approximate function $q_{0}$ of the potential $q$ is given, then it is natural to consider the following m-parametric inverse optimization spectral problem: given a potential $q_{0}$ and $\left\{\lambda_{i}\right\}_{i=1}^{m}$, $m<+\infty$, find a potential $\hat{q}$ closest to $q_{0}$ in a prescribed norm such that $\lambda_{i}=\lambda_{i}(\hat{q})$ for all $i=1, \ldots, m$.

In the present paper, we study the following 1-parametric variant of this problem: for $k \geqslant 1$ we consider the problem

$P(k)$ : given $\lambda \in \mathbb{R}$ and $q_{0} \in L^{2}(0, \pi)$, find a potential $\hat{q} \in L^{2}(0, \pi)$ such that $\lambda=\lambda_{k}(\hat{q})$ and

$$
\left\|q_{0}-\hat{q}\right\|_{L^{2}}=\inf \left\{\left\|q_{0}-q\right\|_{L^{2}}: \lambda=\lambda_{k}(q), q \in L^{2}(0, \pi)\right\} .
$$

Our main result is as follows

Theorem 1.1. Let $q_{0} \in L^{2}(0, \pi)$ be a given potential, $k \geqslant 1$. Then

$\left(1^{\circ}\right)$ for any $\lambda \in \mathbb{R}$, there exists a solution $\hat{q}$ to the inverse optimization spectral problem $P(k)$.

Furthermore,

$\left(2^{o}\right)$ for $\lambda<\lambda_{k}\left(q_{0}\right)$, there exists a non-zero weak solution $\hat{u}_{\delta}$ of $\left.\left(N P_{\delta}\right)\right|_{\delta=1}$, and for $\lambda>\lambda_{k}\left(q_{0}\right)$, there exists a non-zero weak solution $\hat{u}_{\delta}$ of $\left(N P_{\delta}||_{\delta=-1}\right.$ so that the following explicit formula holds

$$
\hat{q}=q_{0}-\delta \hat{u}_{\delta}^{2} \quad \text { a.e. in }(0, \pi) .
$$

$\left(3^{\circ}\right)$ The solution $\hat{u}_{\delta}(x)$ of $\left(N P_{\delta}\right), \delta= \pm 1$ possesses exactly $k-1$ roots in $(0, \pi)$.

The case $k=1$ with the zero Dirichlet boundary conditions has been studied in our recent papers [5], 6], where we proved the existence and uniqueness of solution $\hat{q}$ in the case $\lambda>\lambda_{1}\left(q_{0}\right)$. Furthermore, in this case, stronger result holds, namely: the uniqueness theorem for $\left.\left(N P_{\delta}\right)\right|_{\delta=-1}$ is satisfied so that $N P_{\delta}||_{\delta=-1}$ possesses a unique positive solution for $\lambda>\lambda_{1}\left(q_{0}\right)$.

Remark 1.1. For $\lambda=\lambda_{k}\left(q_{0}\right)$, problem $P(k)$ becomes trivial since in this case $\hat{q}=q_{0}$.

Remark 1.2. Each eigenfunction $\phi_{k}(q)$ of $\mathcal{L}[q]$, as well as each weak solution $\hat{u} \in W_{0}^{1,2}(0, \pi)$ of $\left(N P_{\delta}\right), \delta= \pm 1$, obeys $A C[0, \pi]$-regularity (see e.g. [10]).

It is worth pointing out the following result, which in itself is notably important.

Lemma 1.1. Let $k \geqslant 1$. Then

(i): for each $\lambda \geqslant \lambda_{k}\left(q_{0}\right)$, problem $\left.\left(N P_{\delta}\right)\right|_{\delta=1}$ has no non-zero weak solution with $k-1$ or less roots in $(0, \pi)$;

(ii): for each $\lambda \leqslant \lambda_{k}\left(q_{0}\right)$, problem $\left(N P_{\delta}||_{\delta=-1}\right.$ has no non-zero weak solution with $k-1$ or more roots in $(0, \pi)$.

Theorem 1.1 and Lemma 1.1 imply the following corollary. 
Corollary 1.1. Assume $q_{0} \in L^{2}(0, \pi)$. Then

$\left(1^{o}\right)$ For each $\lambda \in \sigma_{p}(\mathcal{L}[q])$, nonlinear boundary value problem $\left.\left(\sqrt{N P_{\delta}}\right)\right|_{\delta=1}$ has no non-zero weak solution with $k-1$ or less roots in $(0, \pi)$, and problem $\left.\left(N P_{\delta}\right)\right|_{\delta=-1}$ has no non-zero weak solution with $k-1$ or more roots in $(0, \pi)$.

$\left(2^{o}\right)$ For each $\lambda \in\left(\lambda_{k-1}\left(q_{0}\right), \lambda_{k}\left(q_{0}\right)\right), k \geqslant 1$, nonlinear boundary value problem $\left.\left|N P_{\delta}\right|\right|_{\delta=1}$ possesses an infinite sequences of distinct weak solutions $\left(u_{\delta}^{l}\right)_{l=k}^{\infty}$. Moreover, $u_{\delta}^{l}, l=k, \ldots$ has exactly $l-1$ roots in $(0, \pi)$.

$\left(3^{\circ}\right)$ For each $\lambda \in\left(\lambda_{k}\left(q_{0}\right), \lambda_{k+1}\left(q_{0}\right)\right), k \geqslant 1$, nonlinear boundary value problem $\left.\left(N P_{\delta}\right)\right|_{\delta=-1}$ possesses at least $k$ distinct weak solutions $\left(u_{\delta}^{l}\right)_{l=1}^{k}$. Moreover, $u_{\delta}^{l}, l=1, \ldots, k$ has exactly $l-1$ roots in $(0, \pi)$.

Here we assumed that $\lambda_{0}\left(q_{0}\right)=-\infty$.

We emphasize that this result is nothing more than a generalization of the well known Sturm nodal theorem to the nonlinear problem. To the best of the authors' knowledge, such method of proving this statement was not explored previously.

The paper is organised as follows. Section 2 contains some preliminaries and the proof of Lemma 1.1. In Section 3, we prove Theorem 1.1.

\section{PRELIMINARIES}

In what follows, we denote by $\langle\cdot, \cdot\rangle$ and $\|\cdot\|_{L^{2}}$ the scalar product and the norm in $L^{2}(0, \pi)$, respectively; $W^{1,2}(0, \pi), W^{2,2}(0, \pi)$ are usual Sobolev spaces with the norms

$$
\|u\|_{1}=\left(\int_{0}^{\pi}|u|^{2} d x+\int_{0}^{\pi}\left|u^{\prime}\right|^{2} d x\right)^{\frac{1}{2}}, \quad\|u\|_{2}=\left(\int_{0}^{\pi}|u|^{2} d x+\int_{0}^{\pi}\left|u^{\prime \prime}\right|^{2} d x\right)^{\frac{1}{2}} .
$$

$W_{0}^{1,2}:=W_{0}^{1,2}(0, \pi)$ is the closure of $C_{0}^{\infty}(0, \pi)$ in the norm

$$
\|u\|_{1}=\left(\int_{0}^{\pi}|\nabla u|^{2} d x\right)^{\frac{1}{2}}
$$

In what follows, we assume that $\left\|\phi_{k}(q)\right\|_{L^{2}}=1, k=1,2, \ldots$

Proposition 2.1. Let $k \geqslant 1$ and the sequences $\left(q_{j}\right)_{j=1}^{\infty}$ in $L^{2}(0, \pi)$ and $\left(\left|\lambda_{k}\left(q_{j}\right)\right|\right)_{j=1}^{\infty}$ in $\mathbb{R}$ are bounded. Then the sequence $\left(\phi_{k}\left(q_{j}\right)\right)$ is bounded in $W^{2,2}(0, \pi)$.

Proof. We observe that the equation $\mathcal{L}[q] \phi_{k}\left(q_{j}\right)=\lambda_{k}\left(q_{j}\right) \phi_{k}\left(q_{j}\right)$ is equivalent to the following integral equality

$$
\phi_{k}\left(q_{j}\right)(x)=\lambda_{k}\left(q_{j}\right) \int_{0}^{\pi} G_{0}(x, \xi) \phi_{k}\left(q_{j}\right)(\xi) d \xi-\int_{0}^{\pi} G_{0}(x, \xi) q_{j}(\xi) \phi_{k}\left(q_{j}\right)(\xi) d \xi,
$$

where $G_{0}(x, \xi)$ is the integral kernel of operator $(\mathcal{L}[0])^{-1}$. Since $G_{0} \in C[0, \pi] \times C[0, \pi]$, identity (2.1) implies

$$
\left\|\phi_{k}\left(q_{j}\right)\right\|_{C[0, \pi]} \leqslant\left(\Lambda_{k} \max _{\xi, x}\left|G_{0}(x, \xi)\right|+\max _{\xi, x}\left|G_{0}(x, \xi)\right|\left\|q_{j}\right\|_{L^{2}(0, \pi)}\right)\left\|\phi_{k}\left(q_{j}\right)\right\|_{L^{2}(0, \pi)},
$$

where $\Lambda_{k}=\sup _{j}\left|\lambda_{k}\left(q_{j}\right)\right|$. Now taking into account that the set $\left\|q_{j}\right\|_{L^{2}(0, \pi)}$ is bounded, we get

$$
\left\|\phi_{k}\left(q_{j}\right)\right\|_{C[0, \pi]}<C\left\|\phi_{k}\left(q_{j}\right)\right\|_{L^{2}(0, \pi)}
$$

for some $C<+\infty$ independent of $j=1,2, \ldots$. Since $\mathcal{L}[q] \phi_{k}\left(q_{j}\right)=\lambda_{k}\left(q_{j}\right) \phi_{k}\left(q_{j}\right)$, we hence get

$$
\begin{aligned}
\int_{0}^{\pi}\left|\phi_{k}^{\prime \prime}\left(q_{j}\right)\right|^{2} d x & \leqslant \int_{0}^{\pi}\left|q_{j}(x) \phi_{k}\left(q_{j}\right)\right|^{2} d x+\Lambda_{k} \int_{0}^{\pi}\left|\phi_{k}\left(q_{j}\right)\right|^{2} d x \\
& \leqslant\left\|\phi_{k}\left(q_{j}\right)\right\|_{C[0, \pi]}^{2} \int_{0}^{\pi}\left|q_{j}(x)\right|^{2} d x+\Lambda_{k} \int_{0}^{\pi}\left|\phi_{k}\left(q_{j}\right)\right|^{2} d x<C_{1}<+\infty
\end{aligned}
$$


where $C_{1}$ is independent of $j=1,2, \ldots$. Hence, in view of that $\left\|\phi_{k}\left(q_{j}\right)\right\|_{L^{2}}=1$, we obtain

$$
\left\|\phi_{k}\left(q_{j}\right)\right\|_{W^{2,2}}<C_{2}<+\infty
$$

where $C_{2}$ does not depend on $j=1,2, \ldots$

Lemma 2.1. If $B$ is a bounded set in $L^{2}$, then the family of operators $\mathcal{L}[q]$ is uniformly below semi-bounded on $L^{2}$ with respect to $q \in B$, i.e.,

$$
-\infty<\mu \leqslant \inf _{q \in B} \inf \left\{\langle\mathcal{L}[q] \psi, \psi\rangle: \quad \psi \in L^{2}, \quad\|\psi\|_{L^{2}}=1\right\} .
$$

Proof. We follow an approach proposed by Shkalikov in $[9]$. We write $\mathcal{L}[q] y=\mathcal{L}[0] y+Q y$, where $\mathcal{L}[0] y=-y^{\prime \prime}(x), Q y=q(x) y(x)$. Let $a>0$ be a sufficiently large number. We introduce $R(a):=(\mathcal{L}[0]+a I)^{-1 / 2}$. Let us estimate the norm of the operator $R(a) Q R(a)$. For arbitrary $f, g \in L^{2}(0, \pi)$, we have

$$
\langle R(a) Q R(a) f, g\rangle=\langle Q R(a) f, R(a) g\rangle .
$$

Denote by $\mu_{l}=(l)^{2}, \psi_{l}(x)=\sin (l x), l=1,2, \ldots$ the eigenvalues and eigenfunctions of the operator $\mathcal{L}[0]$. Then $f=\sum_{i=1}^{\infty} f_{i} \psi_{i}(x), g=\sum_{j=1}^{\infty} g_{j} \psi_{j}(x)$ in $L^{2}$ and

$$
\langle Q R(a) f, R(a) g\rangle=\sum_{i=1}^{\infty} \sum_{j=1}^{\infty} \frac{g_{j} f_{i}}{s_{i}(a) s_{j}(a)}\left\langle Q \psi_{i}, \psi_{j}\right\rangle,
$$

where $s_{l}(a)=\sqrt{\mu_{l}+a}, l=1,2, \ldots$ We observe that

$$
\left|\left\langle Q \psi_{i}, \psi_{j}\right\rangle\right| \leqslant \max _{x \in[0, \pi]}\left|\psi_{i}(x)\right| \max _{x \in[0, \pi]}\left|\psi_{j}(x)\right| \int_{0}^{\pi}|q(s)| d s<C\|q\|_{L^{2}}, \quad i, j=1,2, \ldots,
$$

where $C<\infty$ does not depend on $i, j=1,2, \ldots$ Hence,

$$
\begin{aligned}
|\langle R(a) Q R(a) f, g\rangle| & \leqslant \sum_{i=1}^{\infty} \sum_{j=1}^{\infty} \frac{\left|g_{j}\right|\left|f_{i}\right|}{s_{i}(a) s_{j}(a)}\left|\left\langle Q \psi_{i}, \psi_{j}\right\rangle\right| \\
& \leqslant C\|q\|_{L_{2}} \sum_{i=1}^{\infty} \sum_{j=1}^{\infty} \frac{\left|g_{j}\right|\left|f_{i}\right|}{s_{j}(a) s_{i}(a)} \leqslant C\|q\|_{L_{2}} \sqrt{\sum_{j=1}^{\infty}\left|g_{j}\right|^{2}} \sqrt{\sum_{i=1}^{\infty}\left|f_{i}\right|^{2}}\left(\sum_{i=1}^{\infty} \frac{1}{\left(s_{i}(a)\right)^{2}}\right) \\
& =C \rho(a)\|q\|_{L_{2}}\|f\|_{L_{2}},
\end{aligned}
$$

where

$$
\rho(a)=\sum_{i=1}^{\infty} \frac{1}{\left(s_{i}(a)\right)^{2}}
$$

Therefore,

$$
|\langle R(a) Q R(a) f, g\rangle| \leqslant C \rho(a)\|q\|_{L_{2}}\|f\|_{L_{2}} \quad \text { for all } \quad f, g \in L^{2} .
$$

We denote $h=R(a) v$ for $v \in L^{2}(0, \pi)$. Since $\|q\|_{L_{2}}$ is bounded on $B$ and $\rho(a) \rightarrow 0$ as $a \rightarrow+\infty$, we obtain that for sufficiently large $a$ the relations

$$
\langle(\mathcal{L}[0]+a I+Q) h, h\rangle=\langle v, v\rangle+\langle R(a) Q R(a) v, v\rangle>0
$$

hold for all $v \in L^{2}$. Hence, for sufficiently large $a$ and for any $\psi \in L^{2}$ such that $\|\psi\|_{L^{2}}=1$, there holds

$$
\langle\mathcal{L}[q] \psi, \psi\rangle>-a\langle\psi, \psi\rangle=-a>-\infty, \quad \text { for all } \quad q \in B .
$$

This completes the proof.

Lemma 2.1 implies immediately the following corollary.

Corollary 2.1. If $B$ is a bounded set in $L^{2}$, then $\lambda_{1}(q) \geqslant \mu>-\infty$ for all $q \in B$, where $\mu$ is independent of $q \in B$. 
Lemma 2.2. For $k \geqslant 1$, the map $\lambda_{k}(\cdot): L^{2}(0, \pi) \rightarrow \mathbb{R}$ is continuously differentiable with the Fréchet-derivative

$$
D \lambda_{k}(q)(h)=\frac{1}{\left\|\phi_{k}(q)\right\|_{L^{2}}^{2}} \int_{0}^{\pi} \phi_{k}^{2}(q) h d x, \quad \text { for all } \quad q, h \in L^{2} .
$$

Proof. Since $\lambda_{k}(q)$ is isolated, Corollary 4.2 in [7] implies that $\lambda_{k}(q)$ is Fréchet differentiable and (2.3) holds. By the analyticity property (see [8]), the map $\phi_{k}(\cdot): L^{2}(0, \pi) \rightarrow W^{2,2}(0, \pi)$ is analytic. Due to the Sobolev theorem, the embedding $W^{2,2}(0, \pi) \subset L^{4}(0, \pi)$ is continuous. Hence, the map $\phi_{k}(\cdot): L^{2}(0, \pi) \rightarrow L^{4}(0, \pi)$ is continuous and therefore the norm of the derivative $D \lambda_{1}(q)$ depends continuously on $q \in L^{2}(0, \pi)$. This implies that $\lambda_{k}(q)$ is continuously differentiable in $L^{2}(0, \pi)$.

Proof of Lemma 1.1. We shall give the proof only for (i). The proof of (ii) is similar.

Let $\lambda \geqslant \lambda_{k}\left(q_{0}\right), k \geqslant 1$ and $\delta=1$. We prove by contradiction, namely, we assume that $\left.N P_{\delta}\right)$ has a non-zero weak solution $u$ with $k-1$ or less roots in $(0, \pi)$. We consider the identities

$$
\begin{aligned}
& u^{\prime \prime}+\left(-q_{0}+\lambda+u^{2}\right) u=0, \\
& \phi_{k}^{\prime \prime}+\left(-q_{0}+\lambda_{k}\right) \phi_{k}=0
\end{aligned}
$$

We observe that $-q_{0}+\lambda+u^{2}>-q_{0}+\lambda_{k}$. However, by the Sturm Comparison Theorem this yields that $u$ should has more than $\phi_{k}$ roots in $(0, \pi)$ that is more than $k-1$ roots in $(0, \pi)$. This is a contradiction.

\section{Proof of the MAIN RESUlT}

We give the proof only for the case $k=2$; the other cases can be proved in the same way. For the proof in the case $k=1$ and $\lambda>\lambda_{1}\left(q_{0}\right)$ see also [5], [6].

Let $\lambda^{*} \in \mathbb{R}$. We consider the following minimization problem

$$
\hat{Q}_{\lambda^{*}}=\inf \left\{Q(q): \lambda^{*}=\lambda_{2}(q), q \in L^{2}(0, \pi)\right\},
$$

where $Q(q):=\left\|q_{0}-q\right\|_{L^{2}}^{2}, q \in L^{2}(0, \pi)$.

Let $q_{j} \in L^{2}(0, \pi), j=1,2, \ldots$ be a minimizing sequence for this problem, i.e., $\lambda_{2}\left(q_{j}\right)=\lambda^{*}$ and $Q\left(q_{j}\right) \rightarrow \hat{Q}_{\lambda^{*}}$. We observe that if $\left\|q_{j}\right\|_{L^{2}}^{2} \rightarrow+\infty$, then $\left\|q_{0}-q_{j}\right\|_{L^{2}}^{2} \rightarrow+\infty$, i.e., $Q(q)$ is a coercive functional. Hence, the sequence $q_{j}$ is bounded in $L^{2}(0, \pi)$, and by the Banach-Alaoglu theorem there exists a subsequence, which we again denote by $\left(q_{j}\right)$, such that $q_{j} \rightarrow \hat{q}$ as $j \rightarrow \infty$ weakly in $L^{2}(0, \pi)$ for some $\hat{q} \in L^{2}(0, \pi)$.

We consider the sequences of eigenfunctions $\left(\phi_{1}\left(q_{j}\right)\right)$ and $\left(\phi_{2}\left(q_{j}\right)\right)$. By assumption, $\lambda^{*}=$ $\lambda_{2}\left(q_{j}\right)$ for all $j=1,2, \ldots$. Furthermore, in view of that $q_{j}$ is bounded in $L^{2}(0, \pi)$, by Corollary 1.1 we infer that the sequence $\lambda_{1}\left(q_{j}\right)$ is bounded below. Therefore, since $\lambda_{1}\left(q_{j}\right)<\lambda_{2}\left(q_{j}\right)=\lambda^{*}$, for $j=1,2, \ldots$, we conclude that $\left|\lambda_{1}\left(q_{j}\right)\right|$ is bounded. It follows from Proposition 2.1 that the sequences $\phi_{1}\left(q_{j}\right)$ and $\phi_{2}\left(q_{j}\right)$ are bounded in $W^{2,2}(0, \pi)$. In view of this, by the Sobolev embedding theorem there exist subsequences, which we again denote by $\phi_{1}\left(q_{j}\right)$ and $\phi_{2}\left(q_{j}\right)$, such that

$$
\phi_{1}\left(q_{j}\right) \rightarrow \phi_{1}^{*}, \quad \phi_{2}\left(q_{j}\right) \rightarrow \phi_{2}^{*} \quad \text { as } \quad j \rightarrow+\infty
$$

strongly in $W^{1,2}(0, \pi)$ and $C^{1}[0, \pi]$ for some $\phi_{1}^{*}, \phi_{2}^{*} \in W_{0}^{1,2}(0, \pi) \cap C^{1}[0, \pi]$. We notice that, since $\left.\left.\| \phi_{1}\left(q_{j}\right)\right)\left\|_{L^{2}}=1,\right\| \phi_{2}\left(q_{j}\right)\right) \|_{L^{2}}=1$, for every $j=1,2, \ldots$, it follows that $\phi_{1}^{*}, \phi_{2}^{*} \neq 0$. Furthermore, we may assume, by passing to a subsequence if necessary, that $\lambda_{1}\left(q_{j}\right) \rightarrow \lambda_{1}^{*}$ as $j \rightarrow \infty$ for some $\lambda_{1}^{*} \in \mathbb{R}$.

Let $m=1,2$. Then

$$
\phi_{m}\left(q_{j}\right)=\lambda_{m}\left(q_{j}\right) \int_{0}^{\pi} G_{0}(x, \xi)\left(\phi_{m}\left(q_{j}\right)(\xi)-\phi_{m}^{*}(\xi)\right) d \xi-\int_{0}^{\pi} G_{0}(x, \xi) q_{j}(\xi)\left(\phi_{m}\left(q_{j}\right)(\xi)-\phi_{m}^{*}(\xi)\right) d \xi
$$




$$
+\lambda_{m}\left(q_{j}\right) \int_{0}^{\pi} G_{0}(x, \xi) \phi_{m}^{*}(\xi) d \xi-\int_{0}^{\pi} G_{0}(x, \xi) q_{j}(\xi) \phi_{m}^{*}(\xi) d \xi
$$

for each $j=1,2, \ldots$ Hence, strong convergences $(3.2)$ and the weak convergence $q_{j} \rightarrow \hat{q}$ in $L^{2}(0, \pi)$ imply

$$
\phi_{m}^{*}(x)=\lambda_{m}^{*} \int_{0}^{\pi} G_{0}(x, \xi) \phi_{m}^{*}(\xi) d \xi-\int_{0}^{\pi} G_{0}(x, \xi) \hat{q}(\xi) \phi_{m}^{*}(\xi) d \xi, \quad m=1,2,
$$

and therefore,

$$
-\frac{d^{2}}{d x^{2}} \phi_{m}^{*}(x)+\hat{q}(x) \phi_{m}^{*}(x)=\lambda_{m}^{*} \phi_{m}^{*}(x), \quad x \in(0, \pi), \quad m=1,2 .
$$

This means that $\left(\lambda_{1}^{*}, \phi_{1}^{*}\right)$ and $\left(\lambda_{2}^{*}, \phi_{2}^{*}\right)$ coincide with some eigenpairs of the operator $\mathcal{L}[\hat{q}]$, i.e.,

$$
\lambda_{m}^{*}=\lambda_{i_{m}}(\hat{q}), \quad \phi_{m}^{*}=\phi_{i_{m}}(\hat{q}), \quad m=1,2,
$$

for some $i_{1}, i_{2} \in \mathbb{N}$. Let us show that $i_{m}=m$ for $m=1,2$. By the Sturm comparison theorem (see e.g.,[10]) for each $j=1,2, \ldots$, every eigenfunction $\phi_{m}\left(q_{j}\right)(x), m=1,2$ has exactly $m-1$ roots. By strong convergences $(3.2)$ in $C^{1}[0, \pi]$ this yields that the limiting function $\phi_{m}^{*}$ has at most $m-1$ roots. Hence, we get that $i_{2} \leqslant 2$ and $i_{1}=1$, i.e., $\lambda_{1}^{*}=\lambda_{1}(\hat{q})$ is the principal eigenvalue of $\mathcal{L}[\hat{q}]$.

Since $\left\langle\phi_{1}\left(q_{j}\right), \phi_{2}\left(q_{j}\right)\right\rangle=0$ for all $j=1,2, \ldots$, by passing to the limit we have $\left\langle\phi_{1}^{*}, \phi_{2}^{*}\right\rangle=0$. Hence, $\phi_{1}^{*} \neq \phi_{2}^{*}$ and therefore,

$$
i_{2}=2, \quad \lambda_{2}^{*}=\lambda_{2}(\hat{q}) .
$$

Thus, $\hat{q}$ is an admissible point for minimization problem (3.1). Taking into consideration that the weak convergence $q_{j} \rightarrow \hat{q}$ in $L^{2}$ imply

$$
Q(\hat{q}) \leqslant \hat{Q}_{\lambda^{*}}
$$

we obtain that $Q(\hat{q})=\hat{Q}_{\lambda^{*}}$. Therefore, $\hat{q}$ is a solution of 3.1 . This concludes the proof of assertion $\left(1^{\circ}\right)$ in Theorem 1.1 .

Let us prove $\left(2^{o}\right)$. Assume that $\lambda^{*} \neq \lambda_{2}\left(q_{0}\right)$.

Since $Q$ and $\lambda_{2}(\hat{q})$ are $C^{1}$-functionals in $L^{2}$, the Lagrange multiplier rule implies

$$
\mu_{1} D Q(\hat{q})(h)+\mu_{2} D \lambda_{2}(\hat{q})(h)=0, \quad \forall h \in L^{2},
$$

where $\mu_{1}, \mu_{2}$ such that $\left|\mu_{1}\right|+\left|\mu_{2}\right| \neq 0$. By $(2.3)$ we therefore get

$$
\int_{\Omega}\left(-2 \mu_{1}\left(q_{0}-\hat{q}\right)+\mu_{2} \phi_{2}^{2}(\hat{q})\right) h d x=0 \quad \text { for all } h \in L^{2},
$$

where $\left\|\phi_{2}(\hat{q})\right\|_{L^{2}}=1$. Hence,

$$
2 \mu_{1}\left(q_{0}-\hat{q}\right)=\mu_{2} \phi_{2}^{2}(\hat{q}) \quad \text { a.e. in } \quad \Omega .
$$

We observe that $\mu_{1} \neq 0, \mu_{2} \neq 0$. Indeed, if $\mu_{1}=0$, then $\phi_{2}(\hat{q})=0$ a.e. in $\Omega$, which is a contradiction. Suppose $\mu_{2}=0$, then $q_{0}=\hat{q}$ a.e. in $\Omega$ and consequently $\lambda^{*}=\lambda_{2}\left(q_{0}\right)$, which contradicts our assumption $\lambda^{*} \neq \lambda_{2}\left(q_{0}\right)$. Hence, we have

$$
\hat{q}=q_{0}-\nu \phi_{2}^{2}(\hat{q}) \text { a.e. in } \Omega,
$$

with some constant $\nu \neq 0$. Substituting this into the identity

$$
-\phi_{2}^{\prime \prime}(\hat{q})+\hat{q} \phi_{2}(\hat{q})=\lambda^{*} \phi_{2}(\hat{q})
$$

we obtain

$$
-\phi_{2}^{\prime \prime}(\hat{q})+q_{0} \phi_{2}(\hat{q})=\lambda^{*} \phi_{2}(\hat{q})+\nu \phi_{2}^{3}(\hat{q}) .
$$

This means that $\hat{u}=|\nu|^{\frac{1}{2}} \phi_{2}(\hat{q})$ satisfies $\left.N P_{\delta}\right)$ and $\hat{q}=q_{0}-\delta \hat{u}^{2}$ a.e. in $\Omega$ with $\delta=\operatorname{sign}(\nu)$. In view of Lemma 1.1. we infer that $\delta=1$ if $\lambda<\lambda_{k}\left(q_{0}\right)$, and $\delta=-1$ if $\lambda>\lambda_{k}\left(q_{0}\right)$. This concludes the proof of $\left(2^{\circ}\right)$. 
The proof of $\left(3^{\circ}\right)$ follows immediately since $\hat{u}(x)=\phi_{k}(\hat{q})(x) \cdot\|\hat{u}\|_{L^{2}}$ and by Sturm nodal theorem the eigenfunction $\phi_{k}(\hat{q})(x)$ of $\mathcal{L}[\hat{q}]$ has exactly $k-1$ roots.

Proof of Corollary 1.1. For $\lambda=\lambda_{k}\left(q_{0}\right), k=1, \ldots$, by (i) in Lemma 1.1 we conclude that nonlinear boundary value problem $\left.\left(N P_{\delta}\right)\right|_{\delta=1}$ has no non-zero weak solution with $k-1$ or less roots in $(0, \pi)$, whereas by (ii) in Lemma 1.1, problem $\left.\left(N P_{\delta}\right)\right|_{\delta=-1}$ has no non-zero weak solution with $k-1$ or more roots in $(0, \pi)$ and this implies $\left(1^{\circ}\right)$.

Since $\lambda_{1}(q)<\lambda_{2}(q)<\ldots$, Assertions $\left(2^{o}\right),\left(3^{o}\right)$ immediately follow Assertions $\left(2^{o}\right),\left(3^{o}\right)$ in Theorem 1.1.

\section{BIBLIOGRAPHY}

1. V. Ambarzumian. Über eine frage der eigenwerttheorie // Zeit. Physik A Hadrons and Nuclei. 53:9, 690-695, (1929).

2. G. Borg. Eine umkehrung der Sturm-Liouvilleschen eigenwertaufgabe // Acta Math. 78:1, 1-96, (1946).

3. D.E. Edmunds, W.D. Evans. Spectral theory and differential operators. 15. Clarendon Press, Oxford (1987).

4. I.M. Gel'fand, B.M. Levitan. On the determination of a differential equation from its spectral function // Izv. RAN. Ser. Matem. 15:4, 309-360, (1951) (in Russian).

5. Y.Sh. Ilyasov, N.F. Valeev. On an inverse optimization spectral problem and a related nonlinear boundary value problem // Matem. Zam. 104:4, 621-625, (2018). [Math. Notes. 104:4, 601-605 (2018).]

6. Y.Sh. Ilyasov, N.F. Valeev. On nonlinear boundary value problem corresponding to $N$-dimensional inverse spectral problem // J. Diff. Equat., to appear.

7. M. Möller, A. Zettl. Differentiable dependence of eigenvalues of operators in Banach spaces // J. Oper. Theor. 36:2, 335-355, (1996).

8. J. Pöschel, and E. Trubowitz. Inverse spectral theory. 130. Pure Appl. Math. Academic Press, Boston (1987).

9. A.A. Shkalikov. Perturbations of self-adjoint and normal operators with discrete spectrum // Uspekhi Matem. Nauk. 71:5, 113-174 (2016). [Russ. Math. Surv 71:5, 907-965 (2016).]

10. A. Zettl. Sturm-Liouville theory. Math. Surv. Monog. 121. Amer. Math. Soc., Providence, RI (2005).

Yavdat Ilyasov

Institute of Mathematics,

Ufa Federal Research Center, RAS,

450008, Ufa, Russia

Instituto de Matemática e Estatística.

Universidade Federal de Goiás,

74001-970, Goiania, Brazil

E-mail: ilyasov02@gmail.com

Nurmukhamet Valeev

Institute of Mathematics,

Ufa Federal Research Center, RAS,

450008, Ufa, Russia

Bashkir State University,

450076, Ufa, Russia

E-mail: valeevnf@mail.ru 